\title{
PRELIMINARY IDEAS FOR A PROJECT ON CULTURAL HERITAGE:"HEVA"- DIGITAL RESOURCES OPTIMIZATION FOR THE ENHANCEMENT OF CULTURAL HERITAGE
}

\author{
Fernández Martín, J.J.[a], García Fernández, J.[a], Delgado del Hoyo, F.J., [b], Finat Codes, J. [b], \\ juanjo@ega.uva.es, jorge.garcia.fernandez@uva.es,frandelhoyo@gmail.com, jfinat@agt.uva.es \\ [a] Laboratory of Architectural Photogrammetry, H.T. College of Architecture, University of Valladolid \\ [b] MoBiVaP, Modeling, Biomechanics, Advanced Visualization Heritage Group. University of Valladolid
}

\begin{abstract}
KEYWORDS: Data Transmission, Cultural Heritage, Digital Resources, Photogrammetry, Virtual Reconstruction, Interoperability
\end{abstract}
\begin{abstract}
:
Cultural Heritage documentation by itself is meaningless if it does not help to create wealth and provide values to society. In recent years, the number of digital contents related to cultural heritage resources is growing in a way that it very difficult to discover reliable information. Thanks to the Internet they can be easily published and distributed but there are three main problems: 1) the quality of the resources is not well evaluated or tagged; 2) the resources are fragmented across several non-linked repositories; 3) most of the resources are not adapted to different kinds of devices and users. These problems are more remarkable in point clouds and three dimensional models digitalized at high resolution, to achieve a higher level of detail because they are too heavy for visualization, transmission and representation.

The present statement of intentions aims to develop a project -HEVA: HEritage VAlue- for creating an effective methodology to simplify and improve the exploitation and transmission of cultural heritage documentation in the three most relevant fields: culture, education and economy. The main goal of the project is to reformulate the objective of cultural heritage documentation from a sustainable perspective, linking the efforts to the achievements and optimizing the processes. At the same time, the project will create synergies between multiple agents involved in documentation, allowing an intelligent diffusion of cultural heritage such that heritage can reach interested people, people who really want to be reached.
\end{abstract}

\section{INTRODUCTION}

From the early nineties there has been a very active policy involving Cultural Heritage issues in the EU. A recent short survey is given in [1]; a more extended presentation can be read in [2]. Furthermore constructive and technological aspects, some crucial issues involving the whole chain value knowledge transfer, educational training and a sustainable management. In the current economic crisis, these issues have increased their relative importance in view of their social impact (including transferability), economic impact (for specialized jobs linked to $\mathrm{CH}$ activities) and policy impact, especially in countries of the Mediterranean basin, where a very large amount of $\mathrm{CH}$ is concentrated. The current economic crisis has transformed social interactions and their sensitivity to exchange processes where economic calculation evaluates the property for his extraction cost rather than replacement cost. The emphasis on its extraction and production of new content / products against recovery and recycling of existing ones, increase the distance from the behavior of civilization against different models of sustainability.

Cultural Heritage is a very important part of European quality of life and increasingly valued by the international community. The teaching-learning relationship on Heritage not only generates a conscience about the needs of the proper management of values and assets, but also encourages collective development processes, setting the stage for continued economic-cultural-educational growth and enrichment. In addition, it provides a support for integrated approaches to environment to maintain natural and historic resources and to improve the management of heritage sites. Thus, it contributes in a very meaningful way to add social and economic value to cultural assets.
The economic value refers to the capability of promoting and providing a support for economic activities around the existence / loss of a known patrimony such as virtual tourism (which is then translated into actual tourism) and the virtual reconstruction (which lets you overlay additional content on the existing assets). Much rethinking is needed as to how 3D models can provide a support for inserting additional contents linked to web services in order to create wealth while posing a precise documentation of heritage, able of stimulating the demand of $\mathrm{CH}$ assets and promoting Heritage Tourism.

The educational value refers to the enrichment of knowledge which can be achieved with digital documentation of heritage at different levels (from school children to scientists) and at different layers. Currently there are a lot of books, pictures, videos, 3D models, etc., which in the current situation need to be reorganized in an appropriate manner and linked to a common digital object. The project HEVA: HEritage VAlue tries to answer to the following question: How can we perform the transfer of multiple meanings of the object from digital assets? 3D models tend to forget the essence of each item and stay at a formal level without other contextual information. HEVA is intended to assess the documentation to label with enriched contents to complete documentation suitable for education.

The cultural value refers to the possibility of knowing, not only the local culture, but other cultures or spread their own, also. The artistic language barrier makes it difficult for the general public access to distant patrimony. The project HEVA: HEritage VAlue alleges that the documentation of heritage help spread our culture beyond their physical boundaries by means of images, models and symbolic representations. It intends to redefine the objectives of heritage documentation to create wealth, increase global culture and to understand from primary education the essence of heritage. 


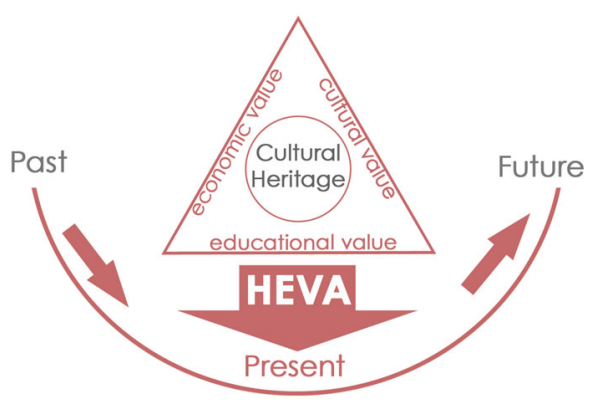

Figure 1: Management Plan for Cultural Heritage in HEVA Project

The Management Plan for Cultural Heritage in HEVA: HEritage Value, shown in Figure 1, is a response to the current economic crisis for a model of enhancement the heritage of a more sustainable view (see Figure 1). For example, if we search Google 3D Warehouse the term "castle" get more than 3000 3D models, this raises the question of whether they are actually effectively used or enjoyed are the result of individual effort without additional incidence for the whole community interested in $\mathrm{CH}$ assets.

The experimental study of the processes of cultural heritage management, with emphasis on research associated with the functional standard methodology, involves the acquisition of new knowledge from the underlying fundamentals, allowing a better understanding of the past, and thoroughly, our present to improve the future. In other words, 3D modeling is not a goal by itself; it must be a mean to improve the sustainability in social and cultural policies. Sustainability is understood as a harmonious balance between environmental, social and economic factors. Hence, the system becomes increasingly unbalanced and thus unstable for future generations if the economic component is not sufficiently valorised, bringing with it social, educational and cultural components degradation.

\subsection{Motivation / Hypothesis}

The proliferation of multimedia digital documents on patrimony, fragmented across multiple repositories and, nonadapted to different visualization devices, causes a decrease in its value and therefore a lower return of the initial investment. Facilitating the diffusion and reutilization of digital content maximizes the cultural, educational and economic Cultural Heritage, at the same time contributing to a sustainable development model. The research in new approaches involves technologies, tools and algorithms, that facilitate the connection and exchange of digital content and adaptation to user's device, will allow to extend the range of possibilities for the dissemination of tangible cultural heritage. The main goal is to improve the quality of the documentation of heritage, the way it is retrieved and displayed, as well as navigation on related information available on the Web, will increase interest in heritage and its exploitation and transmission to future generations. Our starting hypothesis is that this goal can be achieved from the development of a distributed scheme by sharing data infrastructures and smart software tools for knowledge management in a collaborative environment. This hypothesis involves to (a) the development of Semantic tools for integrating and developing the current data infrastructures, and (b) the articulation of a network of communities of users and developers (in terms of Living Labs, mash-ups) for profiting the benefits of DIMAS integration

\subsection{Aims}

Regarding developer and user communities, the current project pursues as a main objective the simplification and improvement of the current processes for the enhancement and dissemination of Cultural Heritage. Manual insertion of digital $\mathrm{CH}$ contents is a very cumbersome and high cost task. Hence, it is necessary to develop a bottom-up approach allowing different agents to participate according to a minimal set of rules. One of its main contributions is the definition of a strategy and methodology for assessing the digital documentation of heritage in implementing outreach strategies related to the fields of CEE (Culture-EducationEconomy). Interoperability issues are being solved at the technological level, but not still to the level of management systems involving diffusion and reuse of the available information in web accessible repositories.

As was explained at the beginning of this section, the methodologies currently used for the documentation of heritage are focused on generating a reliable and accurate documentation, but not in its diffusion. This project will research new technologies, especially those related to the Future Internet, that can help in different aspects of diffusion processes such as reliability, the discovery of related content, transmission and representation (see figures 2 and 3). These issues involve to the articulation between Information and Management Systems supported by the Documentation System.

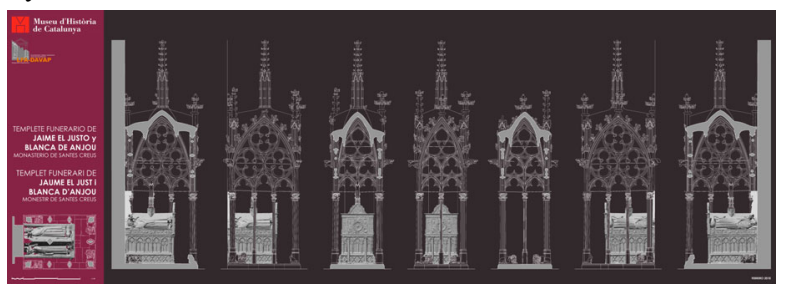

Figure 2: Generation of documentation for diffusion: Documentation of the Royal Pantheons of Santes Creus Monastery for the Museum of Cataluña (Barcelona) Spain

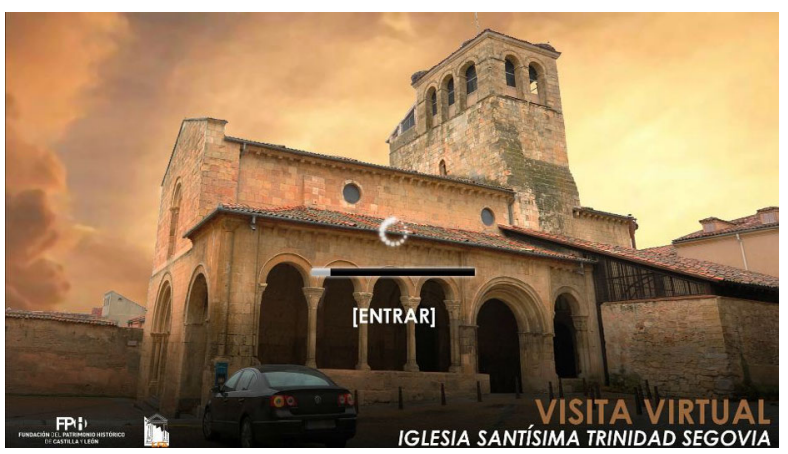

Figure 3: Generation of documentation for diffusion: Virtual Tour of Holy Trinity Church in Segovia, Spain

\section{ANTECEDENTS AND CURRENT STATUS OF KNOWLEDGE}

The digital documentation of heritage consists in capturing real information of objects with high patrimonial value and stores it in digital format [4] [5]. For making reuse easier, the documentation must be performed according to protocols and stored according to key words

Once the contents have been generated, they enter in a cycle of life [6] which must be conveniently exploited to recover 
the initial investment. The information generated is stored as byte stream that can only be interpreted by programs that generate and is not suitable for transmission (because of its high weight) and for integration / reuse in diffusion processes. Therefore it is necessary that in a second phase describes the content in metadata terms so that they can be interpreted and used not only by humans but also by machines without the need of inspecting their contents. The lowest level recommended by the EU is given by DCS (Dublin Core Standards). Their adoption is a first step for guaranteeing the interoperability and the reuse of materials contained in digital repositories.

The generation of metadata and annotation / labeling of the content may be done manually or automatically according to general principles of semantic approaches [7]. Automatic labeling needs advanced expert systems able of recognizing digital contents. Semiautomatic recognition includes to design and implement query, localization, extraction, annotation, classification and indexing procedures.

Once the data has been properly recognized, it is necessary to proceed to the management phase of digital content. Along this stage the documentation is stored in repositories that are currently isolated. This makes it difficult the recovery and discovery of digital content as it is not accessible from an external position. Currently, the solutions are based only on the conventional Web 2.0, allowing to close the cycle with the development of recycling and exploitation of digital contents.

Over this known basis, this project intends to advance in the current knowledge regarding various scientific and technological issues related to the architectural heritage documentation, among which include:

- Topography and Digital Photogrammetry, which allows digitize the objects to turn them into digital content easier to transmit, nevertheless the problems linked to the preservation of digital archives [14]. Today, thanks to new technologies, improved accuracy and cost, it is possible to have a cheaper generation of $\mathrm{CH}$ contents from different $3 \mathrm{D}$ reconstruction hybrid techniques based on a combination of images correspondence from multiple views, laser scanning technology based on time of flight, phase difference and triangulation, etc. [18].

- Computer Graphics and Computational Geometry, are the disciplines that study the representation of 3D models in 2D/3D visualization devices and management of meshes at different resolutions and in-memory representation structures [22]. Related contributions of HEVA Project include a universal display platform, and the detection of the shape and automatic simplification of 3D models to be adapted to visualizers.

- Future Internet which is understood in the sense of involved technologies, with special attention to the development of Web 3.0. The development of this framework for $\mathrm{CH}$ digital contents makes it possible to discover, publish and communicate the digital documentation of heritage already available, as well as that subsequently generated. Some of the technologies involved are: the Semantic Web, including the use of ontologies [16], the Web Services for connecting different repositories of documentation [20], and Web Media that allows to adapt, simplify and transmit in real-time the documentation, especially the 3D models. The application of these technologies to the heritage will enable to obtain a new structural framework based on a Web Oriented Architecture or WOA [21]. Using this architecture it is possible to abstract the recovery and visualization of the contents of the type of device that uses the end user.

\subsection{Current status of related research groups}

At the national level (Spain), the main research groups related to the heritage documentation, are the Heritage Interpretation Laboratory (UAM), the Photogrammetry and Laser Scanner Research Group (UPV), the Laboratory of Geometric Heritage Documentation (EHU) and the Group of Historiography and Andalusian heritage (USe). Currently, its research focuses especially on improving and cheapening the photogrammetric documentation of the assets, so that expected results are related with the same problems which are addressed in this project. Other institutions, laboratories, and interpretation of heritage groups, which are working with $\mathrm{CH}$ digital documents, continue even without addressing the problem of diffusion of the generated documentation in an effective way, which often ends isolated and neglected for being difficult to maintain.

Internationally there are several groups in Europe that are already addressing the goals of this project, although the results are still partial. FORTH-ICSS group (Greece) and CGV, TU-Graz (Austria) are developing an infrastructure or environment, which combine information retrieval and management of digital content. The ISTI-CNR Italian Institute addresses the same problem, only applied on document images of ancient texts. The Semantic Computing Research Group (Finland) has developed technologies and applications within the Semantic Web for heritage management, leaving aside the content adaptation and annotation, which are performed manually. They are the most actively involved in the maintenance of the CIDOC CRM ontology using a semantic browser.

For the development of the present project, it is essential to incorporate RTD groups with expertise in Photogrammetry, Computer Vision, Computer Graphics and Semantic Web. The Laboratory of Architectural Photogrammetry (LFA) and Modeling, Biomechanics, Advanced Visualization and $\mathrm{CH}$ (MoBiVAP) Group, both from the University of Valladolid, subscribers of the current project accredited a rigorous participation in the proposed topic with more than 40 publications and 30 projects / contracts in the last 5 years. Also, both groups have worked on other projects of applied and fundamental research whose results motivate the research proposed for HEVA: HEritage VAlue. Next, we shall comment some of recent achievement performed by this Spanish cluster.

The project MAPA (Models and Algorithms for the Architectural Heritage) was focused on the digitization of patrimony and the technologies that would enable a more accurate and reliable documentation. The main result of this project was a framework for generating accurate and realistic 3D documentation, combining information from different digital sources. The project ADISPA (Analysis, digitization and interoperability in the Architectural Heritage) raised the problem of connecting different tools, repositories and agents involved in both the digitalization and diffusion processes (see Figure 4). The results of this project (still in progress) show the need for a deeper research of new methodologies 
and technologies to simplify the reuse of digital content resulting from the documentation of heritage.

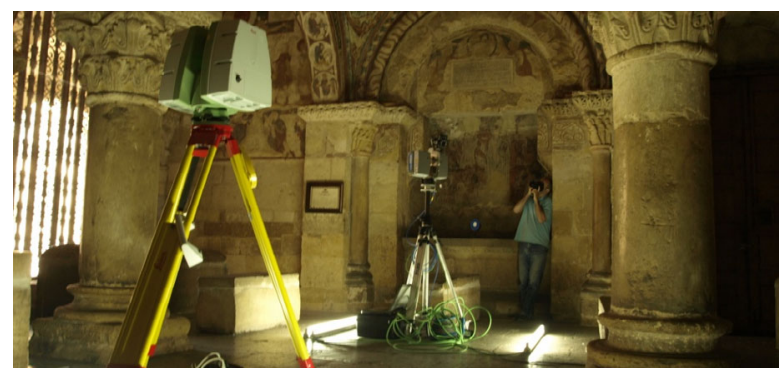

Figure 4: Different physical tools show different digital content. Church of San Isidoro, Spain, Project ADISPA.

Finally, the results of PATRAC Project (Accessible Cultural Heritage) showed that physical spaces and digital documentation of heritage can be accessed from multiple devices to many types of users, and provide a support for advanced interaction as web services in complex $\mathrm{CH}$ environments. This support has been developed with a special attention to persons with motor disabilities (devices mounted on a wheelchair) in a monitored environment A natural extension of this approach includes advances in technologies related to Future Internet, which are of great interest to explore new ways to transmit and communicate the value of heritage through the Web.

The above antecedents allow the involved groups to put in practice previous knowledge and expertise. Innovations and relevance of HEVA: HEritage VAlue objectives are related to the creation of an infrastructure or framework to facilitate the integration, the transfer of knowledge and interaction between different agents involved in the Heritage diffusion . This infrastructure will bring benefits to local and regional institutions (such as a political assistant to maintenance and restoration), to companies related to task analysis, rehabilitation, visualization and diffusion and academic entities themselves. To achieve this, it raises the following specific guidelines:

1. Develop a methodology for assessing the quality of documentation based on the peer review mechanisms.

2. Study on the diffusion current needs of World Heritage in the areas of Education, Culture and Economy

3. Connect documentation from multiple repositories, in multiple formats, enriched with metadata with a shared common vocabulary (ontology)

4. Design and implement algorithms for automatic annotation (metadata extraction) of digital content.

5. Design amd implement algorithms for automatic labeling of parts of 3D models based on recognition of shapes, colors and other geometric and radiometric characteristics of the models.

6. Facilitate simultaneous access to real-time digital documentation, updated from multiple devices using Web standards like HTML 5.

7. Adapt the quality and quantity of digital content to devices and users.

8. Evaluate the improvement in Heritage diffusion as educational, Cultural and Economic products, by reusing digital content.

\section{THE PROJECT}

\subsection{Directing the research}

The research is based on the current context within the documentation of Cultural Heritage. Consistency with the various institutional and government policies (national and international) will be another tool that will promote the conservation of historical heritage through the diffusion. Currently, there exist a lot of official directives and recommendations contained in different charts (Lisbon, London) arising from the European Commission which have been specified in strategic objectives of several Framework Programs involving Construction and ICT areas. These strategic objectives are linked to Strategic Research Agendas (SRA) of Technological Platforms which provide a common support for developing a public-private collaboration with an important incidence at national and European level. These initiatives have been grouped in a JPI (Joint Programming Initiative) on Cultural Heritage and Global Change: a new challenge for Europe" which extends these goals to the 2020 Horizon. An important goal is to promote the research and innovation in $\mathrm{CH}$ involving the whole value chain, increasing the impact, the mobilization and engagement of different stakeholders in a shared environment.

A crucial technology issue included in HEVA Project is to try to solve the interoperability issues concerning management. This solution improves the sustainability of $\mathrm{CH}$ assets because it allows to incorporate available resources in a common semantic framework which is managed by words and sentences of ordinary language. It allows to incorporate advanced software tools (contents extraction, recognition) provides a support for developing new techniques (as the linked to Virtual Tourism as previous to a physical visit), and allows the incorporation of large amount of digital contents in a collaborative framework. In particular, in HEVA: HEritage VAlue we develop a combination of Reconstruction and Recognition tools managed by a semantic framework, which facilitate and cheapen the processes of digitization, retrieval and diffusion. Let us remark that tourism industry generates an estimate annual revenue of EUR 335 billion at EU level, and many of the 9 million jobs in the Tourism sector are linked to $\mathrm{CH}$ [3]. Thus, the return to citizen policies is warranted because heritage tourist activity is an important source of economic income that occurs, generating direct and indirect revenues and providing added value for their social benefit, even if their effects are not measurable in the shortterm

Although the first needs to technological level arise in the area of heritage documentation, to address a global solution it is necessary to carry out a multidisciplinary research process that involves different disciplines. The major research groups can be classified into three: generators, captors and diffusers. All of them can be interpreted as different stakeholders who need a common and easy-to-use framework for sharing experiences and knowledge through their common language. This layered framework is organized at different levels and with different profiles according to their role:

The generators produce documentation to create wealth and provide support for education and spread of specific cultural values. This group includes educators, archaeologists, historians, etc. The captors provide the processes, technologies and tools for reuse or recycling of the currently existing documentation, integrating it with the newly 
generated. This group includes mathematicians, computer scientists and other technologists. The diffusers endow social impact to the contents, using mechanisms based on new communication networks. This group has reporters, communication experts etc.

\subsection{Basic Structure}

The structure of the work will start from generation of metadata and annotation / labeling of the content. Faced with the existing approaches that address this problem manually, in the project HEVA: HEritage Value, will be studied in depth the intelligent allocation of second level metadata from advanced recognition tools applied to $\mathrm{CH}$ knowledge domains.

The semiautomatic annotation of $3 \mathrm{D}$ objects is another area of research that deserves special attention. Currently there is no format that makes it possible to represent and exchange 3D models described with a generic vocabulary. The project will advance in the development of an open XML-based format for transmission of $3 \mathrm{D}$ models labeled items. In this case the labeling of parts of the model can be addressed automatically using recognition algorithms based on multiple characteristics [8]. The recording of the parts of the model can refine the search results and provide the user a richer experience which must be translated to syntactical constructions for $\mathrm{CH}$ assets. To avoid an excessive generalization, we restrict ourselves to some meaningful examples arising from civil and religious architecture of Middle Age and Renaissance.

To solve the problems posed by the storage of documentation in isolated repositories and limitations that involve the Conventional Web 2.0 solutions (consist of posting links to content in multiple forums [6]), the project HEVA: HEritage VAlue will use the approximation Linked Open Data (LOD) [10] [11] from the Semantic Web in which resources are identified by their URL and annotated with the vocabulary of an ontology.

In this same phase there is the problem of reliability and quality of content retrieved, an inherent problem to information published on the Internet [13]. Within this framework is where the HEVA project proposes the development of a valuation methodology based on peer review of content. At the present the majority of strategies to address this problem do so from the standpoint of the visitor comments, where all are equally important. This methodology will be adapted and improved in this project.

Finally, the last phase of life cycle involves the reuse and exploitation of digital contents. At this stage, $\mathrm{CH}$ contents are recovered and visualized by users on different platforms. While current solutions consist of developing applications for each type of device to display the contents stored in local repositories, in HEVA: HEritage VAlue, will deepen in the use of Web standards like HTML 5 and WebGL [14] to develop a browser of digital content associated with multiplatform heritage documentation, in which the contents are adapted [15] according to the type of device and transmitted in "streaming" in real time [16]. The point clouds are the type of content that presents a more complex research in this area. In the case of $3 \mathrm{D}$ content these will be browsed by the user from the device itself.

\subsection{Development phases}

The general methodology to be followed during the project HEVA follows a bottom-up approach. Our proposal part of the efficient creation of $3 \mathrm{D}$ models as a common reference to add economic, educational and cultural value to cultural assets, with the design and implementation of tools and techniques for valorizing $\mathrm{CH}$ assets in multiple ways, formats and layers. These ambitious goals must integrate Documentation, Information and Management Systems (DIMAS) in a common framework which is given by the Semantic Web. This integration is feasible thanks to the design and implementation of a specific Ontology for $\mathrm{CH}$.

The capture of $\mathrm{CH}$ information, assessment processes and the articulation between Information and Management Systems supported by the Documentation System will be addressed throughout different tasks and processes, supported on previous or on-site acquired documentation of archaeological sites, urban-architectural areas, and industrial centers, generated in the first part of the project.

The final results will be validated by a qualitative and quantitative assessment of the number of accesses and the consultation time of the digital documentation of heritage in a national pilot experiment. It will also include subjective assessments of experts in each areas in which the Heritage diffusion is essential (culture, economy, education). These assessments enable a review of the impact that the project results have on the community of actors involved in equity, as well as the achievement degree of the project objectives. The process is developed under the following phases:

Ph1. - Study and collection of requirements associated with Heritage diffusion. It attempts to capture the real needs of the different types of end users that exploit the added value of digital documentation of heritage. In addition, will be scheduled the documentation of different patrimonial scenarios that will be used as test data for the experiments in the following tasks.

Ph2. - Elaboration of a methodology for evaluating the quality of the documentation: It will improve the mechanisms and procedures currently used for the valuation of digital content and, by extension, the documentation of heritage for supporting additional contents (information and management systems).

Ph3: Creating Standards for Heritage documentation from the principle of maximum efficiency: The application of new technologies compatible with the needs of the context and objectives in the field of heritage diffusion will consider modeling quality products, as well as economic / time costs.

Ph4. - Designing an infrastructure for content publication: The distribution and current organization of documentary heritage repositories follows a structure of silos, which makes it difficult the integration in real time. The use of Web technologies to establish a WOA architecture in which contents are interrelated and are automatically published without waiting for its indexation (as happens with Web browsers 2.0) can help to solve this problem. Thus, the documentation can be accessible by reusing existing content.

Ph5. - Designing algorithms for the labeling and automatic semantic annotation: The aggregation of metadata about the documents is a very expensive task, 
especially when these are great data volumes of ancient documents. The automatic realization is far from being perfect, so there is still much space for improvement. Main contribution concern to algorithms for automatic discovery of metadata, as well as automatic labeling of meaningful regions of $3 \mathrm{D}$ models instead if treating them as a whole. This is especially useful for complex façades, buildings or large urban area that has been rebuilt with different styles and technologies.

Ph6. - Designing of easier end-user applications for the adaptation of content to the device: Getting better dissemination of heritage by enhancing the perceived user experience. To make this entirely satisfactory it is necessary that digital content that can be recovered, can be easily adapted to preferences and characteristics of each device (especially to the display and its processing capacity).

Ph7. - Developing a browser and a multiplatform viewer of digital content: Integrating infrastructure resulting from the task S3, content annotated with the results of the S4 and algorithms adaptation and recommendation of the S5it is possible get a Web environment that can be used from multiple device types, regardless of underlying operating system. The result of this task will simplify the access and diffusion of heritage documentation to the different actors involved in the process that will have access to the latest information simultaneously and in real time.

Ph8. - Evaluation, valoration and diffusion of results: The results of the preceding tasks need to be evaluated and validated in order to quantify the degree of fulfillment of project goals. It is able to demonstrate that new technologies help to improve the diffusion and the valorization of patrimony over the current situation, on the other hand presupposes the validation of the hypothesis of the project.

\section{CONCLUSIONS}

Understanding that the text does not respond to a realized project but preliminary ideas, research development marked by the standards and methods set forth herein, allow "putting in value" to the $\mathrm{CH}$ by digitization, interoperability and flow of their assets material and immaterial.

In the scientific-technical section, HEVA: HEritage Value will develop models, tools and technologies for segmentation of 3D models, adaptation and simplification of content to the user and the automatic extraction of metadata from large repositories. In addition the project will enhance and improve the knowledge about the dynamics of the processes of dissemination and exploitation of architectural Cultural Heritage, assessing the perception that users have over the heritage and improving the methodology to assess the quality and reliability of digital documentation.

The results of the project HEVA: HEritage VAlue will contribute, in general, to the improvement of processes for heritage diffusion by developing an open, reusable, standards-based Web, which will be recyclable in different systems of management of digital content.

The presented ideas represent not only an enhancement of the documentation of $\mathrm{CH}$ assets, but will contribute to progress in some challenges involving standardization with obvious implications in interoperability between different systems and sustainability from EEC viewpoint. The achievement of the proposed guidelines will allow to identify the most promising or more deficitary areas will require further study. The need for interaction with techniques and latest design technologies imply a risk of obsolescence as the proposed tasks progress, being necessary periodic evaluation procedures for the adjustment of interests in relation to the evolution of national and international studies/policies involving $\mathrm{CH}$ assets.

From a global viewpoint, the action field of HEVA: HEritage VAlue is defined by mobile margins in different knowledge fields involving digital photogrammetry, computer vision/graphics. Hence, the proposed statement of intentions must be understood as a flux between different communities interested in $\mathrm{CH}$ assets to improve delivery of products, services and governance tools involving cultural, educational and economic resources and according to European directives

\subsection{References}

[1] M.Chapuis and A.Brandt: "Survey and outcomes of Cultural Heritage Research Projects supported in the context of EU environmental research programmers", Directorate General for Research and Innovation, Unit 1.2, EC, 2011.

[2] J.Radic, V.Rajcic and R.Zarnic (eds): "Heritage Protection. Construction Aspects", Intl Conf. Proc., Second HDGK, Dubrovnik, 2006, ISBN 953-95428-1-2

[3] KEA European Affairs, European Commission, 2006.

[4] D. Koller, B. Frischer y G. Humphreys, 2009, «Research challenges for digital archives of 3D cultural heritage models,» Journal on Computing and Cultural Heritage (JOCCH), vol. 2, no 3, p. 7, 2009.

[5] E. Hyvonen, et al, 2009, «Culture Sampo: A National Publication System of Cultural Heritage on the Semantic Web 2.0, " The Semantic Web: Research and Applications, p.851-856, 2009.

[6] F. Remondino, S. Girardi, A. Rizzi y L. Gonzo, 2009, «3D modeling of complex and detailed cultural heritage using multi-resolution data,» Journal on Computing and Cultural Heritage (JOCCH), vol. 2, nº 1, p. 2, 2009.

[7] J. Euzenat y P. Shvaiko, 2007, Ontology matching, Springer-Verlag New York Inc, 2007.

[8] J. Fernández, J. Finat, J. S. José, M. Gonzalo, J. Martínez y L. Fuentes, 2006, «Some recent experiences for cultural heritage sustainability: results and challenges,» Intl workshop on Sustainability in Cultural Heritage (Capri), 2006

\subsection{Acknowledgements}

Professors and researchers of the groups: LFA (Architectural Photogrammetry Laboratory of the University of Valladolid) and MoBiVap (Modeling, Biomechanics, Advanced Visualization Heritage), with a special regard to J.I.SanJoséAlonso, J.Martinez-Rubio and Ruben Martínez-Garcia

Analysis, Digitization and interoperability between systems for architectural heritage, ADISPA, BIA200914254-C02-01BIA2009-14254-C02-01., Dr. Jesús Ignacio San José Alonso, Lab of Architectural Photogrammetry, H.T. School of Architecture, University of Valladolid. 